Article

\title{
A Highly Sensitive, Polarization Maintaining Photonic Crystal Fiber Sensor Operating in the THz Regime
}

\author{
Sohel Rana, Nirmala Kandadai and Harish Subbaraman * \\ Department of Electrical and Computer Engineering, Boise State University, Boise, ID 83725, USA; \\ sohelrana@u.boisestate.edu (S.R.); nirmalakandadai@boisestate.edu (N.K.) \\ * Correspondence: harishsubbaraman@boisestate.edu; Tel.: +1-208-426-4803
}

Received: 21 September 2018; Accepted: 23 October 2018; Published: 25 October 2018

check for updates

\begin{abstract}
In this paper, a high sensitivity, polarization preserving photonic crystal fiber (PCF), based on circular air holes for sensing in the terahertz $(\mathrm{THz})$ band, is presented. The finite element method, a practical and precise computational technique for describing the interactions between light and matter, is used to compute the modal properties of the designed fiber. For the designed PCF, comprising of circular air holes in both the cladding and in the porous core, a relative sensitivity of $73.5 \%$ and a high birefringence of 0.013 are achieved at $1.6 \mathrm{THz}$. The all circular air-hole structure, owing to its simplicity and compatibility with the current fiber draw technique for PCF fabrication, can be realized practically. It is anticipated that the designed fiber can be employed in applications such as detection of biological samples and toxic chemicals, imaging, and spectroscopy.
\end{abstract}

Keywords: far infrared or THz; relative sensitivity; modal birefringence; fiber sensor; photonic crystal fiber

\section{Introduction}

Research in the terahertz $(\mathrm{THz})$ regime, which is the frequency range spanning between $0.1-10 \mathrm{THz}$ in the electromagnetic spectrum, is gaining attention due its promising applications in significant fields such as biosensing, security, imaging, and spectroscopy [1,2]. While the reported works in the literature are based on different technologies [3-10], it is only recently that photonic crystal fibers (PCFs) are being considered for THz sensing applications [11-13]. This is because PCFs offer tremendous design flexibility over the conventional fibers. In PCFs, through the tuning of the parameters of the air holes (pitch, radii of air holes, number of rings, etc.), the waveguiding properties, such as mode properties [14], birefringence [15], dispersion [16], mode confinement [17], and nonlinearity [18], can be effectively controlled. The guiding properties and applications of PCFs are further provided in [19-23].

The research so far on PCF-based THz sensors has focused on reducing the propagation losses in PCFs, as most materials experience a high absorption loss in the terahertz band. Several fiber structures for enabling low-loss propagation have been reported [11-13,24-26]. Of these, porous core based photonic crystal fiber (PCF) sensors [11-13] have shown high sensitivity and low absorption loss. In addition to demonstrating high sensitivity and low absorption loss, maintaining polarization is of great importance in sensing applications to eliminate polarization mode dispersion (PMD), which can change the polarization state during the sensing of analytes, leading to inaccurate sensitivity. An effective approach to maintaining the polarization state in an otherwise symmetric fiber is to break the symmetry in the core or in the cladding, and creating fiber structures with induced high birefringence. With an intention to induce birefringence, rectangular slotted core structures were 
reported in [11,12]. Although the design in [12] showed a high sensitivity of $96.8 \%$ and a high birefringence of 0.0154, the presence of rectangular slots in the core makes these PCFs difficult to realize. Subsequently, a porous core design with elliptical air holes was proposed in [13] to alleviate the fabrication difficulties of the design reported in [12]; however, at a cost of drastically reducing the relative sensitivity to $68.8 \%$. Moreover, it is difficult to maintain conditions during the drawing process that simultaneously preserves circular hole shapes in the cladding and elliptical hole shapes in the core.

With an aim to further improve fabrication feasibility through the incorporation of circular air holes throughout the PCF, and to achieve high sensitivity and birefringence, in this paper, we propose an all circular air hole-based porous core PCF structure. Our simulation results indicate that the design exhibits a high relative sensitivity of $73.5 \%$, high modal birefringence of 0.013 , and low confinement loss. While the relative sensitivity of the proposed fiber is lower than those reported in $[11,12]$, the all circular air hole structure can be handled in the state-of-the-art fiber drawing towers.

\section{Geometric Structure}

In the terahertz regime, every material experiences a certain absorption loss, and for this reason background material choice is very crucial. Cyclic olefin co-polymer, polymethyl methacrylate (PMMA), polycarbonate (PC), high-density polyethylene (HDPE), and polytetrafluoroethylene (PTFE) are all suitable fiber materials due to their low absorption loss in the $\mathrm{THz}$ regime. In this work, we chose cyclic olefin co-polymer, with the trade name TOPAS, because it offered certain advantages over others, including lower absorption loss [27], humidity insensitivity [28], etc. The refractive index of the TOPAS used was 1.5258 [29].

A regular hexagonal PCF structure was modified by scaling down the radius of several air holes in the center of the fiber, to form a porous core PCF structure. The cross section of the proposed fiber is shown in Figure 1. In order to induce birefringence, additional air holes (shown in orange) were introduced in the core to break the symmetry. Symmetry of the cladding was broken by removing four air holes at the top and the bottom corners (shown as red dashed air holes in the outermost ring). The length of the core was denoted by $L$ and its value was chosen as $480 \mu \mathrm{m}$. The center-to-center distance between two adjacent air holes (also called pitch) of the regular hexagonal structure, both in the core and in the cladding, were $\Lambda / 2$ and $\Lambda$, respectively, where $\Lambda=80 \mu \mathrm{m}$. The diameter of the air holes in the cladding (denoted by $d$ ) was chosen as $37.6 \mu \mathrm{m}$. The overall fiber structure diameter was $1056 \mu \mathrm{m}$. To demonstrate sensor operation, similar treatment as in [11-13] for the structure, wherein two analytes (water $(n=1.33)$ and ethanol $(n=1.354))$ were used to fill the air holes in the core, was considered as these analytes are common in our daily life and detection is necessary to avoid their harmful effects.

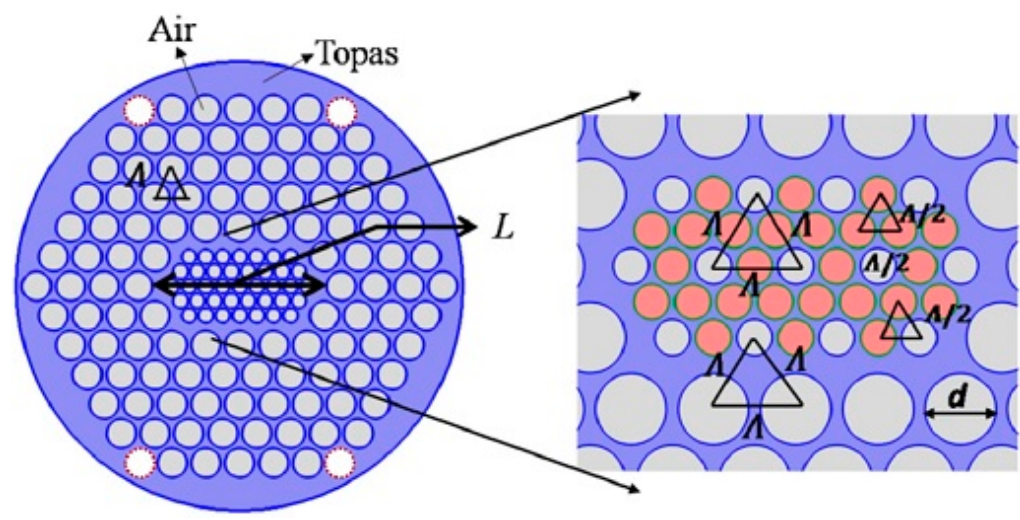

Figure 1. Cross section of the designed fiber (red dashed air holes in the cladding are missing, and orange colors in the core are intermediate air holes introduced to induce birefringence). 


\section{Results and Discussion}

The modal parameters of the designed fiber were computed using an efficient finite element method (FEM) toolset available in COMSOL software. The electric field distribution of the fundamental mode in the fiber is shown in Figure 2 for two orthogonal polarizations at a frequency of $1.6 \mathrm{THz}$. The PCF design parameters in Section 2 were chosen so that at $1.6 \mathrm{THz}$ our design exhibited a maximum relative sensitivity of $73.5 \%$ and a high birefringence of 0.013 .

(a)

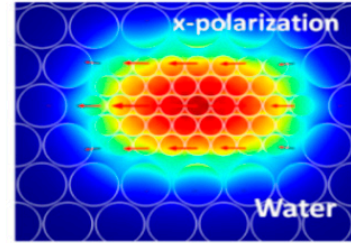

(c)

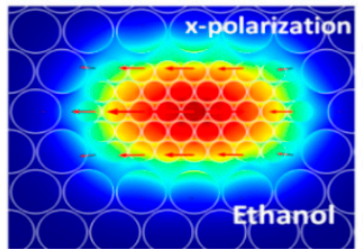

(b)

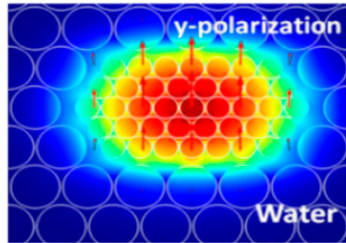

(d)

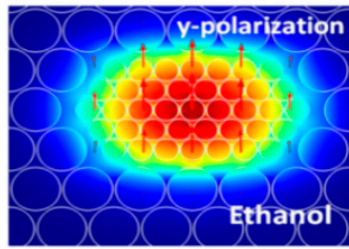

Figure 2. Electric field distribution of the fundamental mode in the proposed fiber at $1.6 \mathrm{THz}$ : (a) $x$-polarization for water-filled core; (b) $y$-polarization for water-filled core; (c) $x$-polarization for ethanol-filled core; and (d) $y$-polarization for ethanol-filled core.

\subsection{Relative Sensitivity}

The extent of the interaction between light and analytes defines the relative sensitivity of the proposed design. The sensitivity of the designed fiber was investigated by calculating the sensitivity coefficient, $r$, which was given by [30]:

$$
r=\frac{n_{r}}{n_{\mathrm{eff}}} \times P
$$

where $n_{r}$ is the refractive index of the analyte to be sensed and $n_{\mathrm{eff}}$ is the effective refractive index of the fiber material. $P$ is the fraction of useful power in the core air holes, which can be calculated using [30]:

$$
P=\frac{\int_{\text {sample }} \operatorname{Re}\left(E_{\mathrm{x}} H_{y}-E_{y} H_{x}\right) d x d y}{\int_{\text {total }} \operatorname{Re}\left(E_{x} H_{y}-E_{y} H_{x}\right) d x d y} \times 100
$$

where, $E_{x}$ and $E_{y}$ are the transverse and the longitudinal electric fields, respectively; and $H_{x}$ and $H_{y}$ are the transverse and the longitudinal magnetic fields, respectively. Higher refractive index material in the core created a large index difference between the core and the cladding, which in turn assisted in stronger confinement, leading to increased interaction of light with the material. Figures 3 and 4 show the relative sensitivity in both $x$ - and $y$-polarizations, when the core air holes were filled with water and ethanol, respectively. The $x$-polarization exhibited comparatively higher sensitivity than the $y$-polarization, which was due to the larger number of air holes, filled with analytes, that light interacted with in the $\mathrm{x}$ direction compared to the $\mathrm{y}$ direction. As can be seen from Figure 3 , the highest relative sensitivity values obtained for $x$-polarization and $y$-polarization when the core air holes were filled with water were $71.5 \%$ and $68 \%$, respectively. From Figure 4 , it can be seen that the highest relative sensitivity values obtained for $x$-polarization and $y$-polarization when the core air holes were filled with ethanol were $73.5 \%$ and $71 \%$, respectively. The obtained relative sensitivities were lower than those reported in [11,12]. This is because the designs in [11,12] are based on noncircular air-holes, both in the core and in the cladding, and they have a higher porosity compared to a strict circular packing structure. The higher porosity leads to increased interaction of light with the analytes, thus increasing the sensitivity of the sensor. However, these noncircular air-hole designs are difficult 
to fabricate using state-of-the-art fiber draw processes. A departure from rectangular slots in the air core was made in [13], wherein elliptical air holes were used in the core. A relative sensitivity of $68.8 \%$ was reported in that structure.

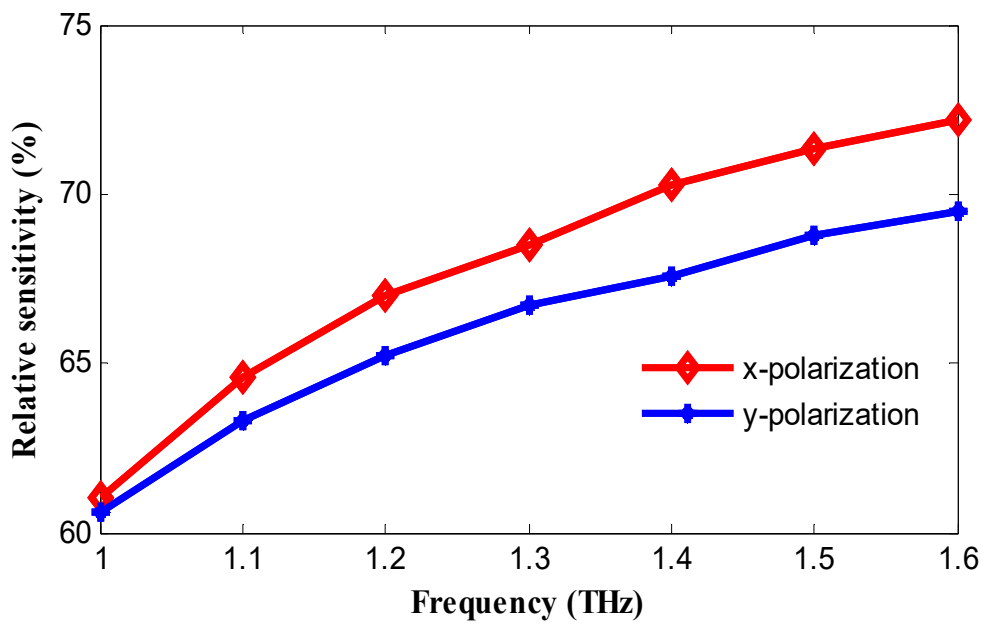

Figure 3. Relative sensitivity as a function of frequency when the core air holes were filled with water.

Due to the higher relative sensitivity in the $x$-polarization state in our designed fiber, the discussion henceforth is focused on $x$-polarization, unless otherwise stated. For our structure, we also considered the effect of dimensional deviation in the air hole radii on the sensitivity, as this can happen during the fiber draw process. We simulated the change in sensitivity when the radius of the core air holes was changed by $\pm 3 \%$. A change in cladding air hole radius was not considered as the mode is tightly confined in the core air holes, and any change in sensitivity would be predominantly affected by the core air holes. It can be seen from Figure 5 that the relative sensitivity for water filled holes changed from $71.5 \%$ to $71.9 \%$ and $69.5 \%$ when the hole radius was varied by $+3 \%$ and $-3 \%$, respectively, whereas when ethanol was used as the analyte, the sensitivity changed from $73.5 \%$ to $73.9 \%$ and $71.1 \%$, respectively. It can be seen that there were no significant changes in the relative sensitivity for the variation of the core air holes radius by $\pm 3 \%$. By enhancing the air filling fraction (AFF) in the core, that is the ratio of air hole diameter to pitch, the relative sensitivity could be increased because it brings the air holes close to one another, which in turn lets sufficient light pass into the core air holes. However, increasing AFF would reduce the index difference between two orthogonal polarizations, leading to a mismatched polarization state.

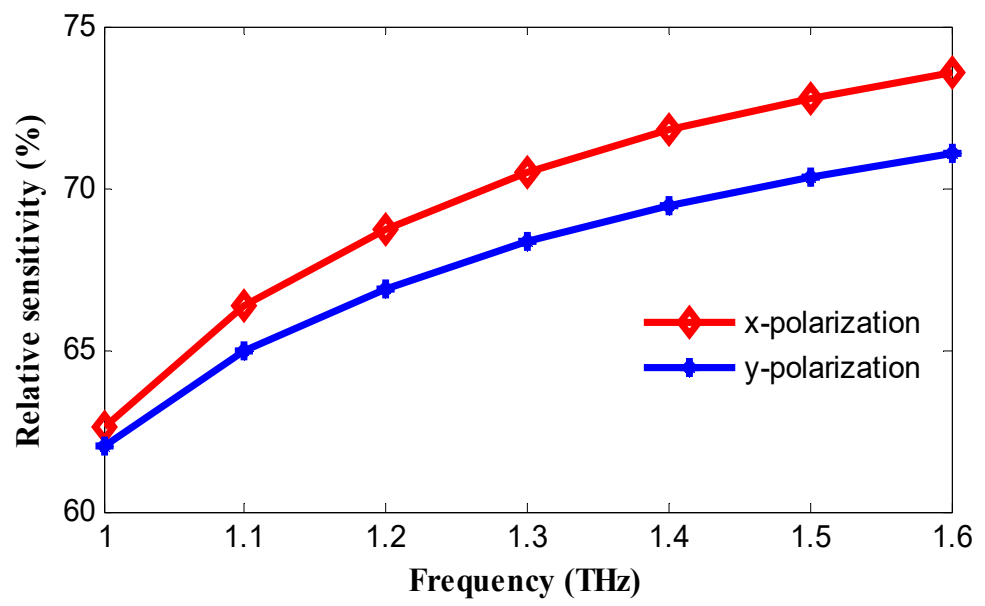

Figure 4. Relative sensitivity as a function of frequency when the core air holes were filled with ethanol. 


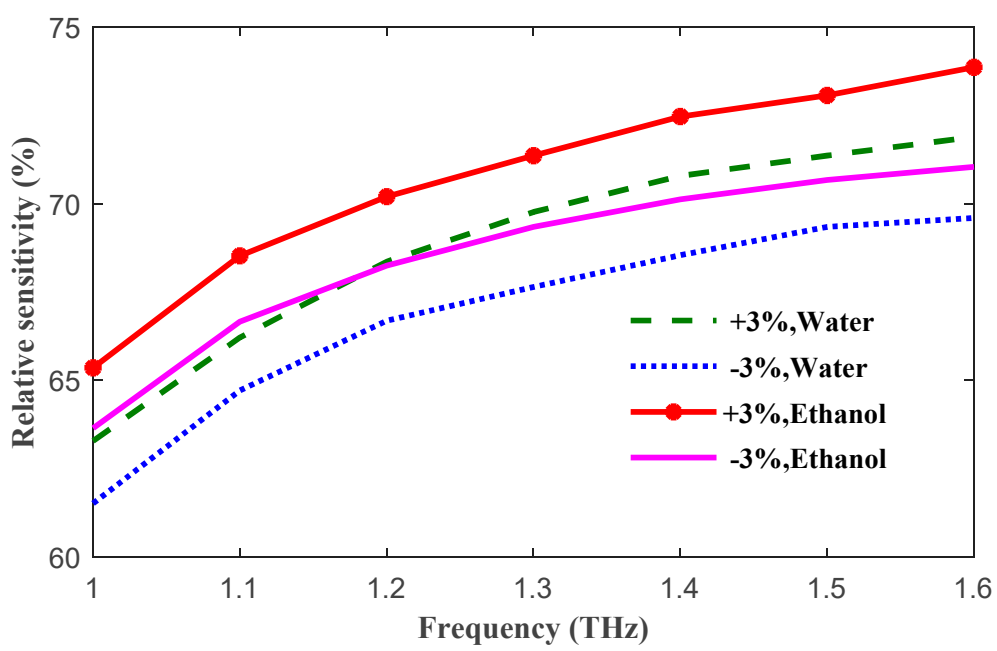

Figure 5. Relative sensitivity of the sensor when the core air hole radius was varied by $\pm 3 \%$ for the water and ethanol cases.

\subsection{Modal Birefringence}

The symmetry of the designed fiber was broken intentionally in order to preserve the polarization state during the interaction between light and analytes. A high birefringence is expected to maintain the polarization state in sensing applications. The modal birefringence can be calculated using:

$$
B=\left|n_{x}-n_{y}\right|
$$

where $n_{x}$ and $n_{y}$ represent the effective refractive indices for the $x$-polarization and the $y$-polarization states, respectively. The calculated modal birefringence is shown in Figure 6 for water-filled core holes (green) and ethanol-filled core holes (pink), from which it can be concluded that water shows higher birefringence than ethanol. The obtained birefringence was higher than that reported in [11], and lower than those reported in $[12,13]$. It is easy to break the symmetry of the core by using elliptical air holes and obtain high birefringence [12]. However, it again makes the fabrication of the structure difficult. The birefringence increased with increasing frequency up to $1.5 \mathrm{THz}$, saturated up to $1.6 \mathrm{THz}$, and then started to decrease beyond $1.6 \mathrm{THz}$ (not shown in Figure). This decrease beyond $1.6 \mathrm{THz}$ was probably due to increased absorption loss, which affected the mode index.

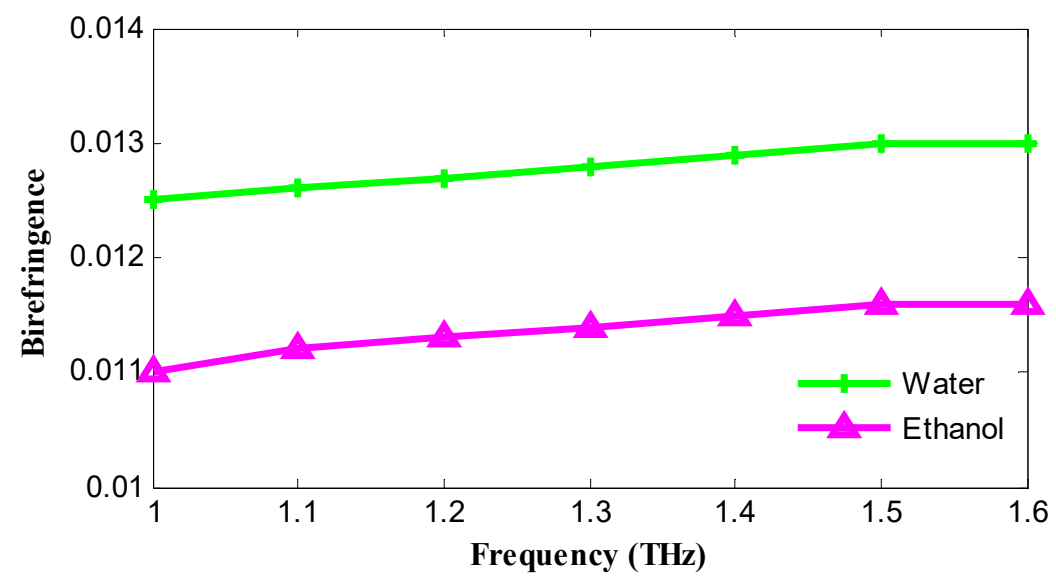

Figure 6. Birefringence of water-filled (green) and ethanol-filled (pink) core hole-based PCF as a function of frequency. 


\subsection{Confinement Loss}

Confinement loss is very important in sensing applications and it occurs due to the finite cladding dimensions. The confinement loss can be calculated from the imaginary part of the effective refractive index using [29]:

$$
L_{C}=8.686 \frac{2 \pi f}{c} \operatorname{Im}\left(n_{\mathrm{eff}}\right)
$$

where $f$ is the frequency, $c$ is the light velocity, and $\operatorname{Im}\left(n_{\text {eff }}\right)$ is the imaginary part of the effective refractive index. Figure 7 shows the confinement loss calculated for both analytes. The higher refractive index difference between the core and the cladding in the case of ethanol-filled core holes led to tighter confinement, compared to that in water-filled core holes, resulting in a lower confinement loss. At higher frequencies, light interacted much more with the material, which in turn resulted in a lower confinement loss. Figure 8 shows the effective refractive index as a function of frequency. As expected, ethanol-filled core hole PCF exhibited a larger effective refractive index compared to water-filled core hole PCF.

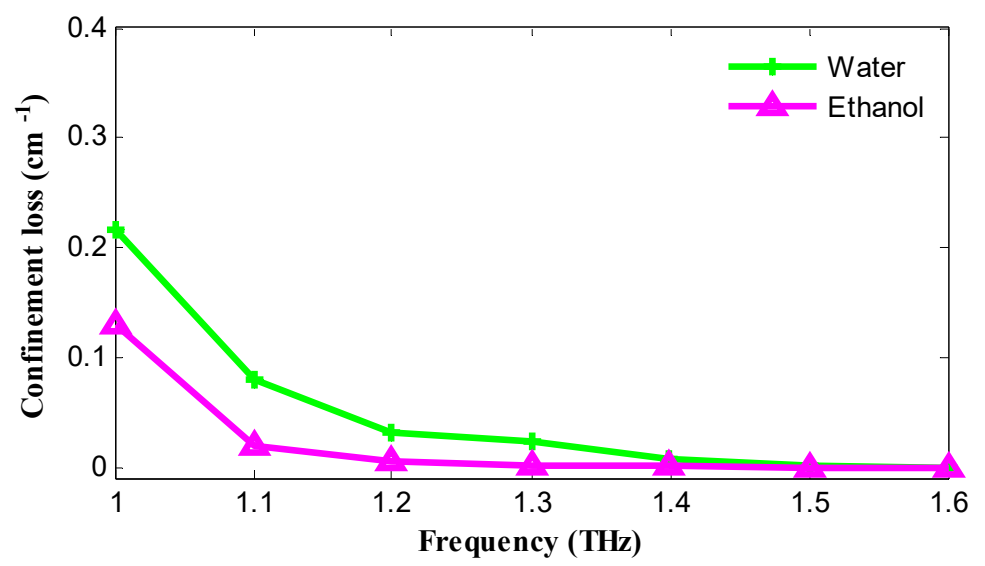

Figure 7. Confinement loss of water-filled (green) and ethanol-filled (pink) core PCF as a function of frequency.

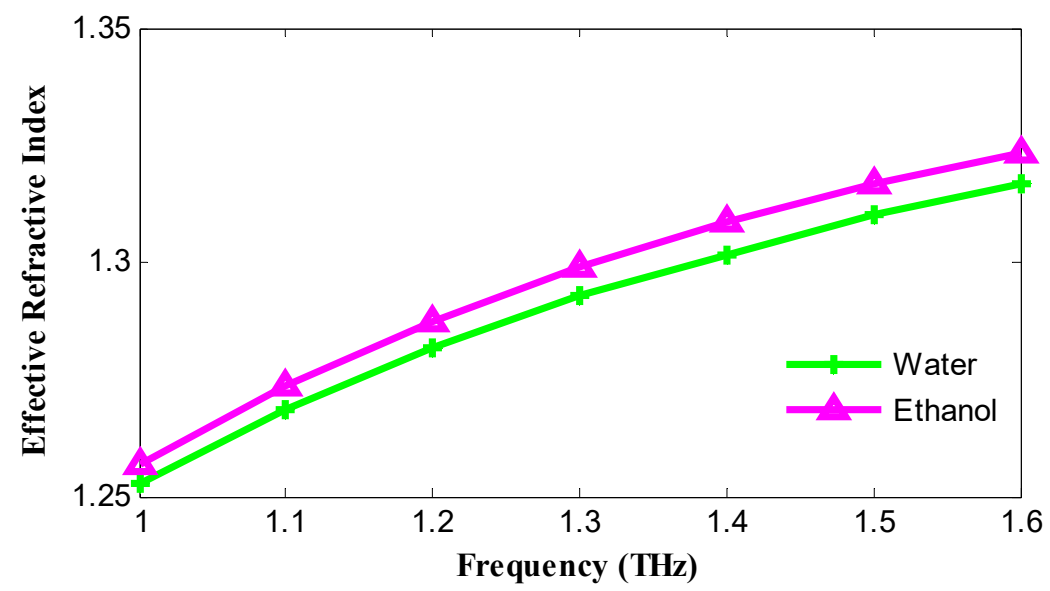

Figure 8. Effective refractive index as a function of frequency.

Table 1 provides a comparison between the designed fiber and others porous core PCF structures reported in literature for $\mathrm{THz}$ sensing. 
Table 1. Comparison of the proposed designed fiber with other reported fibers in the literature for sensing application.

\begin{tabular}{cccccc}
\hline Ref. & Core Air Hole Shape & Cladding Air Hole Shape & Relative Sensitivity & Birefringence & Fabrication Feasibility \\
\hline 11 & Rectangular slot & Kagome structure & $85.7 \%$ & 0.005 & difficult \\
12 & Rectangular slot & Rectangular slot & $96.8 \%$ & 0.0154 & $\begin{array}{c}\text { difficult } \\
\text { difficult } \\
13\end{array}$ \\
Elliptical & Circular & $68.8 \%$ & 0.0176 & compatible with the \\
$\begin{array}{c}\text { Proposed } \\
\text { (this work) }\end{array}$ & Circular & Circular & $73.5 \%$ & 0.013 & state-of-the-art process \\
\hline
\end{tabular}

\section{Fabrication Methods}

Fabrication is an important issue for the practical realization of the fiber sensors. To date, a number of ongoing fabrication methods, such as extrusion, capillary stacking, and stack and drilling are applied to fabricate a wide variety of fiber preforms and structures. High porosity, both in the core and in the cladding, increases the fabrication difficulties of the PCF; however, technologies used for microstructure fibers have made it easy to some extent. A porous fiber was practically realized in [31]. The designs reported in [11,12] used rectangular slotted air holes, whereas elliptical shape air holes are used in [13]. However, difficulties arise during the fabrication of noncircular holes in microstructure fibers due to a lot of reasons, which include the action of surface tension, viscous stresses, heating, and pressure effects during the fiber draw. If the shape of the air holes is not circular in the preform stage, those effects subsequently lead to nonuniformly oriented hole deformations [32]. As our proposed fiber consists only of circular air holes, both in the core and in the cladding, it is expected that the fabrication of the fiber will be achieved using the stack and draw technique [33], or by drilling holes in the preform and then drawing it into a PCF.

\section{Conclusion}

A photonic crystal fiber-based sensor has been proposed for sensing in the terahertz range. High relative sensitivity for both analytes (water and ethanol) has been reported. Furthermore, high modal birefringence and low confinement loss are obtained from the designed fiber. High birefringence preserves the polarization state, whereas low confinement loss provides a stronger interaction between light and the analytes to be sensed. Compared to other porous core PCF THz sensors reported in literature, our design is easily realizable using the state-of-the-art fabrication process, as it involves a single hole shape (circular) in both the core and the cladding.

Author Contributions: S.R. designed and simulated the structure. H.S. helped with writing and reviewing the paper. N.K. helped with writing and reviewing the paper.

Funding: This research received no external funding.

Conflicts of Interest: The authors declare no conflict of interest.

\section{References}

1. Awad, M.M.; Cheville, R.A. Transmission tera-hertzwaveguide based imaging below the diffraction limit. Appl. Phys. Lett. 2005, 86, 221107. [CrossRef]

2. Cook, D.J.; Decker, B.K.; Allen, M.G. Quantitative THz spectroscopy of explosive materials. In Proceedings of the 2005 Optical Terahertz Science and Technology, Orlando, FL, USA, 14-16 March 2005; p. MA6. [CrossRef]

3. Fischer, B.M.; Helm, H.; Jepsen, P.U. Chemical recognition with broadband THz spectroscopy. Proc. IEEE. 2007, 95, 1592-1604. [CrossRef]

4. Fischer, B.M.; Hoffmann, M.; Helm, H.; Modjesch, G.; Jepsen, P.U. Chemical recognition in terahertz time-domain spectroscopy and imaging. Semicond. Sci. Technol. 2005, 20, S246. Available online: stacks.iop. org/SST/20/S246 (accessed on 20 September 2018). [CrossRef]

5. Zhang, J.Q.; Grischkowsky, D. Waveguide terahertz time-domain spectroscopy of manometer water layers. Opt. Lett. 2004, 29, 1617-1619. [CrossRef] [PubMed] 
6. Nagel, M.; Bolivar, P.H.; Brucherseifer, M.; Kurz, H.; Bosserhoff, A.; Bttner, R. Integrated THz technology for label-free genetic diagnostics. Appl. Phys. Lett. 2002, 80, 154-156. [CrossRef]

7. Fischer, B.M. Terahertz time-domain spectroscopy and imaging of artificial RNA. Opt. Express 2005, 13, 5205-5215. [CrossRef] [PubMed]

8. Cheon, H.; Yang, H.J.; Son, J.H. Toward clinical cancer imaging using terahertz spectroscopy. IEEE J. Sel. Top. Quant. Electron. 2017, 23, 1-9. [CrossRef]

9. Zaytsev, K.I.; Kudrin, K.G.; Koroleva, S.A.; Fokina, I.N.; Volodarskaya, S.I.; Novitskaya, E.V.; Perov, A.N.; Karasik, V.E.; Yurchenko, S.O. Medical diagnostics using terahertz pulsed spectroscopy. J. Phys. Conf. Ser. 2014, 486, 012014. Available online: http:/ /iopscience.iop.org/article/10.1088/1742-6596/486/1/012014/ meta (accessed on 20 September 2018). [CrossRef]

10. Kiwa, T.; Kondo, J.; Oka, S.; Kawayama, I.; Yamada, H.; Tonouchi, M.; Tsukada, K. Chemical sensing plate with a laser-terahertz monitoring system. Appl. Opt. 2008, 47, 3324-3327. [CrossRef] [PubMed]

11. Islam, M.S.; Sultana, J.; Ahmed, K.; Islam, M.R.; Dinovitser, A.; Ng, B.W.H.; Abbott, D. A novel approach for spectroscopic chemical identification using photonic crystal fiber in the terahertz regime. IEEE Sens. J. 2018, 18, 575-582. [CrossRef]

12. Islam, M.S.; Sultana, J.; Rifat, A.A.; Dinovitser, A.; Ng, B.W.H.; Abbott, D. Terahertz sensing in a hollow core photonic crystal fiber. IEEE Sens. J. 2018, 18, 4073-4080. [CrossRef]

13. Sultana, J.; Islam, M.S.; Ahmed, K.; Dinovitser, A.; Ng, B.W.H.; Abbott, D. Terahertz detection of alcohol using photonic crystal fiber sensor. Appl. Opt. 2018, 57, 2426-2433. [CrossRef] [PubMed]

14. Akowuah, E.K.; Ademgil, H.; Haxha, S.; Malek, F.A. An endlessly single-mode photonic crystal fiber with low chromatic dispersion, and bend and rotational insensitivity. J. Lightwave Technol. 2009, 27, 3940-3947. [CrossRef]

15. Yue, Y.; Kai, G.; Wang, Z.; Sun, T.; Jin, L.; Lu, Y.; Zhang, C.; Liu, J.; Li, Y.; Liu, Y. Highly birefringent elliptic-hole photonic crystal fibrewith squeezed hexagonal lattice. Opt. Lett. 2007, 32, 469-471. [CrossRef] [PubMed]

16. Saitoh, K.; Koshiba, M.; Hasegawa, T.; Sasaoka, E. Chromatic dispersion control in photonic crystal fibres: Application to ultra-Flattened dispersion. Opt. Express 2003, 11, 843-852. [CrossRef] [PubMed]

17. Liu, S.; Wang, Y.; Hou, M.; Guo, J.; Li, Z.; Lu, P. Anti-resonant reflecting guidance in alcohol-filled hollow core photonic crystal fiber for sensing applications. Opt. Express 2013, 21, 31690-31697. [CrossRef] [PubMed]

18. Hansen, K.P. Dispersion flattened hybrid-core nonlinear photonic crystal fiber. Opt. Express 2003, 11, 1503-1509. [CrossRef] [PubMed]

19. Elbaz, D.; Malka, D.; Zalevsky, Z. Photonic crystal fiber based $1 \times \mathrm{N}$ intensity and wavelength splitters/couplers. Electromagnetics 2012, 32, 209-220. [CrossRef]

20. Malka, D.; Zalevsky, Z. Multicore photonic crystal fiber based $1 \times 8$ two-dimensional intensity splitters/couplers. Electromagnetics 2013, 33, 413-420. [CrossRef]

21. Sintov, Y.; Malka, D.; Zalevsky, Z. Prospects for diode-pumped alkali-atom-based hollow-core photonic-crystal fiber lasers. Opt. lett. 2014, 39, 4655-4658. [CrossRef] [PubMed]

22. Malka, D.; Sintov, Y.; Zalevsky, Z. Fiber-laser monolithic coherent beam combiner based on multicore photonic crystal fiber. Opt. Eng. 2014, 54. [CrossRef]

23. Malka, D.; Cohen, E.; Zalevsky, Z. Design of $4 \times 1$ power beam combiner based on multiCore photonic crystal fiber. Appl. Sci. 2017, 7, 695. [CrossRef]

24. Rana, S.; Rakin, A.S.; Subbaraman, H.; Leonhardt, R.; Abbott, D. Low loss and low dispersion fiber for transmission applications in the terahertz regime. IEEE Photonics Technol. Lett. 2017, 29, 830-833. [CrossRef]

25. Islam, M.S.; Faisal, M.; Razzak, S.M.A. Extremely low loss porous-core photonic crystal fiber with ultra-flat dispersion in terahertz regime. J. Opt. Soc. Am. B 2017, 34, 1747-1754. [CrossRef]

26. Rana, S.; Rakin, A.S.; Hasan, M.R.; Reza, M.S.; Leonhartd, R.; Abbott, D.; Subbaraman, H. Low loss and flat dispersion Kagome photonic crystal fiber in the terahertz regime. Opt. Commun. 2017, 410, 452-456. [CrossRef]

27. Nielsen, K.; Rasmussen, H.K.; Adam, A.J.L.; Planken, P.C.M.; Bang, O.; Jepsen, P.U. Bendable, low-loss TOPAS fibers for the terahertz frequency range. Opt. Express 2009, 17, 8592-8601. [CrossRef] [PubMed]

28. Yuan, W.; Khan, L.; Webb, D.J.; Kalli, K.; Rasmussen, H.K.; Stefani, A.; Bang, O. Humidity insensitive TOPAS polymer fiber Bragg grating sensor. Opt. Express 2011, 19, 19731-19739. [CrossRef] [PubMed] 
29. Rana, S.; Islam, M.S.; Faisal, M.; Roy, K.C.; Islam, R.; Kaijage, S.F. Single-mode porous fiber for low-loss polarization maintaining terahertz transmission. Opt. Eng. 2016, 55, 076114. [CrossRef]

30. Ademgil, H.; Haxha, S. Highly birefringent nonlinear PCF for optical sensing of analytes in aqueous solutions. Optik Int. J. Light Electron Opt. 2014, 125, 6274-6278. [CrossRef]

31. Atakaramians, S.; Afshar, S.; Ebendorff-Heidepriem, H.; Nagel, M.; Fischer, B.M.; Abbott, D.; Monro, T.M. THz porous fibers: Design, fabrication and experimental characterization. Opt. Express 2009, 17, 14053-14062. [CrossRef] [PubMed]

32. Issa, N.A.; van Eijkelenborg, M.A.; Fellew, M.; Cox, F.; Henry, G.; Large, M.C. Fabrication and study of microstructured optical fibers with elliptical holes. Opt. Lett. 2004, 29, 1336-1338. [CrossRef] [PubMed]

33. Pysz, D.; Kujawa, I.; Stępień, R.; Klimczak, M.; Filipkowski, A.; Franczyk, M.; Kociszewski, L.; Buźniak, J.; Haraśny, K.; Buczyński, R. Stack and draw fabrication of soft glass microstructured fiber optics. Bull. Pol. Ac. Tech. 2014, 62, 667-682. [CrossRef]

(C) 2018 by the authors. Licensee MDPI, Basel, Switzerland. This article is an open access article distributed under the terms and conditions of the Creative Commons Attribution (CC BY) license (http:// creativecommons.org/licenses/by/4.0/). 